\title{
Consideración crítica de las adicciones digitales
}

\author{
Xavier Carbonell \\ xaviercs@blanquerna.url.edu \\ Universitat Ramon Llull, España \\ Fran Calvo \\ fran.calvo@udg.edu \\ Universitat de Girona, España \\ Tayana Panova \\ panova1@illinois.edu \\ Universitat Ramon Llull, España \\ Marta Beranuy \\ marta.beranuy@unavarra.es \\ Universidad de Navarra, España
}

\begin{abstract}
Resumen
Este trabajo propone un análisis crítico de las adicciones tecnológicas y se centra, especialmente, en las redes sociales, el móvil y los videojuegos. El uso de las redes sociales no responde al patrón descrito para la adicción puesto que no cumple los criterios establecidos para ella. Puede ser problemático y generar consecuencias negativas, pero éstas son leves y no llegan al nivel de las adicciones. Es más fructífero estudiar el contexto del perfil psicológico del usuario, las motivaciones y gratificaciones y su contexto socio-cultural para entender cómo se produce un uso problemático y cómo combatirlo. La falta de estudios longitudinales y de muestras clínicas invitan a la cautela para no patologizar conductas novedosas. En el caso del teléfono móvil, no consideramos que sea una adicción puesto que el móvil es una plataforma en el que se pueden encontrar contenidos que pueden llegar a ser problemáticos, pero el móvil en sí mismo no es el problema. Sobre los videojuegos cabe mencionar que tanto el DSM-5 como la CIE- 11 lo han considerado un trastorno adictivo que se caracteriza por un patrón de comportamiento de juego persistente y recurrente que conlleva un deterioro o malestar clínicamente significativo en el que es necesario seguir investigando para describir sus particularidades clínicas y mejorar las propuestas de tratamiento.
\end{abstract}

\section{Palabras clave}

redes sociales en línea; trastorno de juego en internet; teléfono inteligente; adicción; DSM-5 


\title{
A critical consideration of digital addictions
}

\author{
Xavier Carbonell \\ xaviercs@blanquerna.url.edu \\ Universitat Ramon Llull, Spain \\ Fran Calvo \\ fran.calvo@udg.edu \\ Universitat de Girona, Spain \\ Tayana Panova \\ panova1@illinois.edu \\ Universitat Ramon Llull, Spain \\ Marta Beranuy \\ marta.beranuy@unavarra.es \\ Universidad de Navarra, Spain
}

\begin{abstract}
This work proposes a critical analysis of technological addictions and focuses especially on social networks, smartphones and video games. The use of social networks sites does not respond to the pattern described for addiction since it does not meet the criteria established for it. It can be problematic and have negative consequences, but these are mild and do not rise to the level of addictions. It is more fruitful to study the context of the user's psychological profile, motivations and gratifications and their socio-cultural context to understand how a problematic use occurs. The lack of longitudinal studies and clinical samples invite caution to avoid pathologizing novel behaviors. In the case of the smartphones, we do not consider it to be an addiction since the mobile phone is a platform where you can find content that can become problematic, but the smartphone itself is not the problem. Regarding video games, it should be mentioned that both the DSM- 5 and the ICD-11 have considered it the internet gaming disorder that is characterized by a persistent and recurrent pattern of gaming behavior that entails a clinically significant deterioration or discomfort; further research is necessary to describe its clinical characteristics and improve treatment proposals.
\end{abstract}

\section{Keywords}

Social network sites; internet gaming disorder; IGD; Smartphone addiction; addiction; DSM-5 


\section{Introducción}

Desde que en 1996 se presentó la comunicación Adicción a Internet: la emergencia de un nuevo trastorno en el congreso de la Asociación Americana de Psicología (Young, 1996), se mantiene viva la discusión sobre la adicción a Internet y a otras tecnologías con cientos de publicaciones al respecto (Carbonell, Guardiola, Beranuy, \& Bellés, 2009; Carbonell, Guardiola, Fuster, Gil, \& Panova, 2016). El interés por la posible adicción a Internet, videojuegos, juegos de rol en línea, televisión y teléfonos móviles ha dado lugar a un nuevo campo de estudio, el de las adicciones tecnológicas (Griffiths, 1995) o digitales (Carbonell, 2020a). De hecho, la adicción a videojuegos figura en la lista de trastornos que merecen más investigación del DSM-5 (American Psychiatric Association, 2013) y en la lista de enfermedades de la Organización Mundial de la Salud (2019).

El objetivo del presente análisis es considerar críticamente el estado de la cuestión sobre las denominadas adicciones tecnológicas (Griffiths, 1995) como redes sociales, teléfono inteligente y videojuegos a partir de una revisión subjetiva de la literatura. Para ello, en el primer apartado se estudian las características esenciales de las adicciones comportamentales. En los siguientes apartados se revisa críticamente la documentación científica sobre redes sociales en línea, teléfono móvil (o smartphone) y videojuegos. El artículo finaliza con una reflexiones y recomendaciones.

\section{a. Las adicciones comportamentales}

Según Goodman (1990), la adicción define una condición por la cual un comportamiento problemático se caracteriza por un fallo recurrente para controlar el comportamiento y por el hecho de seguir con el comportamiento a pesar de las consecuencias negativas significativas. Los síntomas de adicción propuestos por Griffiths $(1995,2005)$ son la modificación del estado de ánimo, la tolerancia, la prominencia, los síntomas de abstinencia, el conflicto y la recaída. Sin embargo, se ha criticado que estas descripciones de los criterios pueden cubrir un amplio espectro de gravedad, que puede ser más o menos clínicamente significativo. Según Carbonell et al. (2008), los elementos diagnósticos esenciales de las adicciones son la dependencia psicológica y los efectos perjudiciales. La dependencia psicológica incluye el deseo, ansia o pulsión irresistible (craving), la polarización o focalización atencional, la modificación del estado de ánimo (sensación creciente de tensión que precede inmediatamente el inicio de la conducta; placer o alivio o incluso euforia mientras se realiza la conducta; agitación o irritabilidad si no es posible llevar a cabo la conducta) y la incapacidad de control e impotencia. Los efectos perjudiciales tienen que ser graves y alterar tanto el ámbito intrapersonal (experimentación subjetiva de malestar) como el interpersonal (trabajo, estudio, finanzas, ocio, relaciones sociales, problemas legales, etc.). Recientemente, Saunders et al. (2017) declararon que en el borrador de la CIE-11, las características principales de la dependencia de sustancias son: a) un fuerte impulso interno para consumir la sustancia, junto con una capacidad deteriorada para controlar ese consumo; b) prioridad al consumo de la sustancia frente a otras actividades; y c) persistencia de su consumo a pesar del daño y las consecuencias adversas. Específicamente, sobre la adicción conductual, Kardefelt-Winther et al. (2017) propusieron una definición de dos componentes: a) deterioro funcional significativo o angustia como consecuencia directa del comportamiento, y b) persistencia en el tiempo.

En la tabla 1 se puede observar el paralelismo de estos síntomas de manera que el análisis de su significado evoca una misma definición teórica que se apoya en dos puntos clave: a) las consecuencias negativas, el daño (grave) o el deterioro ocasionado por la repetición de la conducta problemática y b) la dependencia, tanto psicológica (deseo, focalización y pérdida de control) como física (tolerancia y abstinencia), que conducen a perpetuar la conducta. Por 
lo tanto, a estos dos puntos clave, hay que añadir que este comportamiento persiste en el tiempo y puede ocasionar recaídas.

\begin{tabular}{|c|c|c|c|c|c|c|c|c|}
\hline $\begin{array}{l}\text { Fuent } \\
\text { e }\end{array}$ & $\begin{array}{l}\text { Estado } \\
\text { de } \\
\text { ánimo }\end{array}$ & $\begin{array}{l}\text { Cravi } \\
\text { ng }\end{array}$ & $\begin{array}{l}\text { Toler } \\
\text { ancia }\end{array}$ & $\begin{array}{l}\text { Pérdida } \\
\text { de } \\
\text { control }\end{array}$ & $\begin{array}{l}\text { Focaliza } \\
\text { ción }\end{array}$ & $\begin{array}{l}\text { Abstin } \\
\text { encia }\end{array}$ & $\begin{array}{l}\text { Consecue } \\
\text { ncias } \\
\text { negativas }\end{array}$ & $\begin{array}{l}\text { Recaíd } \\
\text { a }\end{array}$ \\
\hline $\begin{array}{l}\text { Good } \\
\text { man } \\
(1990 \\
)\end{array}$ & & & & $\begin{array}{l}\text { Fallo } \\
\text { recurrente } \\
\text { en el } \\
\text { control del } \\
\text { comporta } \\
\text { miento }\end{array}$ & & & $\begin{array}{l}\text { Continuar } \\
\text { a pesar de } \\
\text { consecuenc } \\
\text { ias } \\
\text { negativas }\end{array}$ & \\
\hline $\begin{array}{l}\text { Griffit } \\
\text { hs } \\
(1995, \\
2005)\end{array}$ & $\begin{array}{l}\text { Modificac } \\
\text { ión del } \\
\text { estado } \\
\text { de } \\
\text { ánimo }\end{array}$ & & $\begin{array}{l}\text { Tolera } \\
\text { ncia }\end{array}$ & & $\begin{array}{l}\text { Prominen } \\
\text { cia }\end{array}$ & $\begin{array}{l}\text { Síntom } \\
\text { as de } \\
\text { abstin } \\
\text { encia }\end{array}$ & Conflicto & $\begin{array}{l}\text { Recaíd } \\
\text { a }\end{array}$ \\
\hline $\begin{array}{l}\text { Carbo } \\
\text { nell et } \\
\text { al. } \\
(2008 \\
\text { ) }\end{array}$ & $\begin{array}{l}\text { Depende } \\
\text { ncia } \\
\text { psicológi } \\
\text { ca: } \\
\text { modifica } \\
\text { ción } \\
\text { estado } \\
\text { ánimo }\end{array}$ & $\begin{array}{l}\text { Depen } \\
\text { dencia } \\
\text { psicol } \\
\text { ógica: } \\
\text { cravin } \\
g\end{array}$ & & $\begin{array}{l}\text { Dependenc } \\
\text { ia } \\
\text { psicológica } \\
: \\
\text { incapacida } \\
\text { d de } \\
\text { control }\end{array}$ & $\begin{array}{l}\text { Depende } \\
\text { ncia } \\
\text { psicológi } \\
\text { ca: } \\
\text { focalizaci } \\
\text { ón }\end{array}$ & & $\begin{array}{l}\text { Efectos } \\
\text { perjudiciale } \\
\text { s graves }\end{array}$ & \\
\hline $\begin{array}{l}\text { Saund } \\
\text { ers et } \\
\text { al. } \\
(2017 \\
\text { ) }\end{array}$ & & $\begin{array}{l}\text { Fuerte } \\
\text { impul } \\
\text { so } \\
\text { para } \\
\text { consu } \\
\text { mir }\end{array}$ & & $\begin{array}{l}\text { Capacidad } \\
\text { deteriorad } \\
\text { a para } \\
\text { controlar } \\
\text { el } \\
\text { consumo }\end{array}$ & $\begin{array}{l}\text { Prioridad } \\
\text { al } \\
\text { consumo } \\
\text { frente a } \\
\text { otras } \\
\text { actividad } \\
\text { es }\end{array}$ & & $\begin{array}{l}\text { Persistenci } \\
\text { a del } \\
\text { consumo a } \\
\text { pesar del } \\
\text { daño }\end{array}$ & \\
\hline $\begin{array}{l}\text { Kardef } \\
\text { elt- } \\
\text { Winth } \\
\text { er et } \\
\text { al. } \\
(2017 \\
\text { ) }\end{array}$ & & & & & & & $\begin{array}{l}\text { Deterioro } \\
\text { significativ } \\
\text { o como } \\
\text { consecuenc } \\
\text { ia del } \\
\text { comportam } \\
\text { iento }\end{array}$ & $\begin{array}{l}\text { Persist } \\
\text { encia } \\
\text { en el } \\
\text { tiempo }\end{array}$ \\
\hline
\end{tabular}

Tabla 1. Análisis comparativo de los síntomas sobre la adicción comportamental aportados por los diferentes autores. 


\section{b. Las redes sociales en línea}

Las redes sociales en línea son aplicaciones tecnológicas interactivas mediadas por dispositivos electrónicos fijos o móviles que facilitan la creación o el intercambio de información, ideas, intereses profesionales y otras formas de expresión a través de comunidades y redes virtuales (Aichner, Grünfelder, Maurer, \& Jegeni, 2020; Boyd, 2014). La popularidad de cada una de las redes sociales depende de factores como el país, la edad y la cultura; algunas de las conocidas en los últimos años son Facebook, WhatsApp, Instagram, Twitter, LinkedIn y Tik Tok. A pesar de sus diferencias, las características comunes de las redes sociales es que permiten conectar personas con intereses comunes e intercambiar información (Aichner et al., 2020).

Las redes sociales son tan populares y se teme que sean adictivas porque son potentes reforzadores. Los seres humanos son fundamentalmente criaturas sociales que buscan tener experiencias sociales regulares y las redes sociales en línea les ofrecen precisamente eso, interacciones instantáneas, fáciles y regulares con una red de contactos sociales. Además, las personas anhelan un sentido de control sobre sus vidas que las redes sociales en línea les proporcionan al permitirles elaborar su perfil y, en consecuencia, ejercer control sobre cómo son percibidos. Respecto a las consecuencias negativas, la capacidad de saber lo que los compañeros están haciendo puede conducir a una comparación social perjudicial, alentar una búsqueda de retroalimentación positiva y validación, y conducir a una menor satisfacción con la vida si uno siente que "no está a la altura" de sus compañeros. La capacidad de controlar la identidad en línea propia puede conducir a discrepancias entre el ser real y el ser idealizado con la consiguiente reducción de la satisfacción vital.

Sin embargo, el potencial adictivo de las redes sociales es controvertido. Algunos investigadores sostienen que los comportamientos y los estados mentales de los usuarios de redes sociales son análogos a los de los adictos a las sustancias y, por lo tanto, las redes sociales deben considerarse adictivas, mientras otros autores argumentan que las consecuencias negativas del uso de las redes sociales no supera el umbral necesario para merecer el diagnóstico de adicción (para una revisión ver Andreassen, 2015; Carbonell \& Panova, 2017; Kuss \& Griffiths, 2011; Ryan, Chester, Reece, \& Xenos, 2014). Independientemente del consenso al que se llegue sobre este tema, cierto es que algunos usos de las redes sociales se asocian a consecuencias negativas de diferentes grados de gravedad que merece la pena estudiar. Como dice Boyd (2014), el foco de preocupación debería ser por qué y cómo estas consecuencias negativas surgen y qué se puede hacer para minimizarlas (Boyd, 2014).

Para entender por qué las personas llevan a cabo actividades particulares en exceso es necesario identificar por qué son reforzadoras. Respecto al uso de las redes sociales, la respuesta simple es que los humanos son criaturas sociales. Durante milenios, hemos vivido en grupos y la interacción humana ha sido vital para nuestra supervivencia, bienestar y prosperidad. Cuando interactuamos con personas que nos gustan, generalmente nos sentimos más felices, seguros y saludables. Cuando no interactuamos con otras personas durante períodos prolongados de tiempo, tendemos a sentirnos solos, un estado negativo que se ha correlacionado con riesgos para la salud como enfermedades cardíacas, derrames cerebrales, depresión e ideación suicida entre otros.. Por lo tanto, es natural buscar interacciones sociales regulares con otras personas. Las redes sociales nos ofrecen tales interacciones de una manera rápida, gratuita, fácil y divertida. En resumen, las redes sociales satisfacen una necesidad humana básica (Carbonell \& Panova, 2017).

De los dos criterios mencionados en la introducción que definen de una forma teórica la adicción, no se cumplen todos los factores de la dependencia psicológica y las consecuencias negativas son leves o moderadas y transitorias. La preocupación sobre la adicción a las redes sociales surge de la observación de que las personas usan esta tecnología durante un 
período considerable de tiempo de forma regular y continúan haciéndolo incluso cuando surgen consecuencias negativas relacionadas con su uso y sacrifican actividades como dormir, estudiar y trabajar (Andreassen \& Pallesen, 2014; Carbonell \& Panova, 2017). Sin embargo, la gravedad de estas consecuencias negativas no es especialmente alta, ni el usuario las entiende siempre como una consecuencia directa de su uso de las redes sociales, por lo que no desencadena los niveles de preocupación necesarios para motivar una disminución en uso (Turel, He, Xue, Xiao, \& Bechara, 2014). Si una persona no cree que las consecuencias estén directamente relacionadas con su comportamiento, no se puede esperar que quiera o intente minimizar ese comportamiento. Por lo tanto, el criterio de "continúa participando en el comportamiento a pesar de las consecuencias negativas" tampoco puede diagnosticar de manera fiable una "adicción" a las redes sociales porque implica una conciencia de las consecuencias negativas del uso de redes sociales (Carbonell \& Panova, 2017).

Se ha de tener en cuenta que la literatura psicológica tiende a patologizar el acto de elegir comportamientos agradables en lugar de escoger comportamientos saludables o productivos. En un análisis típico de costo-beneficio de un inicio de sesión en Facebook o Instagram, los beneficios incluyen ver imágenes interesantes, satisfacer la curiosidad sobre lo que están haciendo sus compañeros y participar en interacciones sociales agradables. Los costes incluyen perder un poco de tiempo que de otra manera podría dedicarse a dormir, estudiar, trabajar $u$ otras actividades productivas, pero no necesariamente agradables. Dada la elección entre trabajar o divertirse, elegir no trabajar no es indicativo de un trastorno, es una preferencia por un placer a corto plazo en lugar de una inversión menos placentera a largo plazo (Carbonell \& Panova, 2017; Panova \& Carbonell, 2018). Lo que es beneficioso para nosotros no siempre es lo que elegimos porque somos propensos a elegir el placer sobre la productividad. Antes de que existieran las redes sociales, esta tendencia se manifestaba con otros distractores. El problema es más notable ahora porque los distractores digitales, como las redes sociales, están muy disponibles y accesibles, lo que facilita ignorar alternativas más saludables o productivas.

Si se utiliza el argumento de que un individuo elige hacer un comportamiento a pesar de las consecuencias negativas para ilustrar el uso problemático, entonces se deben examinar situaciones en las que la consecuencia negativa potencial es lo suficientemente grave. Un buen ejemplo de esto en el contexto de las redes sociales en línea es cuando una persona elige usar las redes sociales mientras conduce. Pese a que existen leyes que prohíben este comportamiento peligroso, se producen accidentes debido a las distracciones causadas por el uso del teléfono. Sin embargo, esto no es necesariamente indicativo de una adicción a las redes sociales. Este comportamiento puede atribuirse a un juicio impreciso de la probabilidad de riesgo y a la incapacidad para activar los niveles requeridos de control de los impulsos, a menudo debido a síntomas de déficit de atención (Turel \& Bechara, 2016). Las personas tienden a usar sus teléfonos mientras conducen cuando creen que tienen el control suficiente del automóvil (tal vez porque están en una ruta familiar, hay poco tráfico, la carretera está despejado, es un viaje directo, están en una semáforo, etc.), cuando están cognitivamente poco estimuladas y sienten que son capaces de ahorrar los recursos de atención. Lo que a menudo pasa, y es lo que causa los accidentes, es que no calculan los factores externos que pueden aparecer repentinamente y requerir una atención inmediata que se está prestando al teléfono. Sin embargo, estos errores de juicio y fallas en el autocontrol pueden existir y existen fuera del marco de la adicción.

Aquellas consecuencias que sí podemos asociar directamente al uso de las redes sociales son leves, mucho más leves que las consecuencias asociadas a las adicciones. Esta es una de las razones por las que la adicción a las redes sociales no se ha incluido en el DSM- 5 ni en la CIE-11 y si lo han sido los videojuegos. Petry y O'Brien (2013), al revisar el proceso de elaboración del DSM-5, argumentaron que incluir condiciones que no causen angustia y 
deterioro significativos (por ejemplo, la adicción al baile o al chocolate) reduce la credibilidad de los trastornos mentales. Además, es muy osado argumentar que una herramienta que satisface la necesidad humana básica de socialización y solo ocasiona consecuencias negativas leves, crea adicción.

La 'patologización' de las nuevas tecnologías se remonta a decenas de años con ejemplos notables que incluyen la radio y la televisión e incluso la imprenta (como en el caso del Quijote). Para determinar si un determinado comportamiento es adictivo, debemos dejar atrás los juicios prematuros sobre qué tan bueno o malo es el comportamiento y explorar sus efectos en la conducta de los usuarios. Y los argumentos contra la existencia de adicción a las redes sociales son múltiples como demuestra la falta de estudios longitudinales y muestras clínicas, la gran probabilidad de falsos positivos y la falta de coherencia en la metodología, la terminología, las puntuaciones de corte y los criterios de diagnóstico (Andreassen, 2015; Calvo, Carbonell, Oberst, \& Fuster, 2018; Carbonell \& Panova, 2017). Estas limitaciones ilustran una falta de validez de constructo para la adicción a las redes sociales (Ryan et al., 2014). Está claro que ciertas personas usan las redes sociales de una manera problemática $y$, en consecuencia, experimentan consecuencias negativas. Sin embargo, etiquetar esta situación como una adicción no nos da mucha información sobre los mecanismos específicos por los cuales las redes sociales pueden conducir a estas consecuencias negativas (Panova, Carbonell, Chamarro, \& Puerta-Cortés, 2020). En este momento, la investigación apoya cuatro motivaciones principales en la raíz del uso problemático de las redes sociales: el efecto novedad, el miedo a quedarse fuera (Fear of Missing Out, FoMO), la búsqueda de validación y la evitación del afrontamiento.

El 'efecto novedad' dificulta determinar si la adicción a las redes sociales es un verdadero trastorno. Éste se produce cuando un nuevo dispositivo o tecnología se usa mucho, principalmente porque es nuevo e interesante, al igual que la forma en que un juguete nuevo recibe toda la atención de un niño durante un corto período de tiempo (Jeno, Vandvik, Eliassen, \& Grytnes, 2019). Cuando el objeto pierde su novedad y su capacidad para cautivar la atención, su uso se normaliza e integra en el funcionamiento normal del usuario. Sin embargo, dado que las redes sociales son relativamente recientes, surgen nuevas cada pocos años y las instauradas se actualizan cada pocos meses, se puede argumentar que el efecto de novedad de las redes sociales todavía es vigente. De los estudios transversales y demográficos podemos extraer que las redes sociales tienen picos de uso durante la adolescencia cuando los adolescentes se conectan por primera vez en línea para desarrollar sus redes sociales e identidades, actividades que son características de su etapa de desarrollo, pero, con la edad, su uso se vuelve menos intenso y menos problemático (Andreassen, 2015; Carbonell, Chamarro, Oberst, Rodrigo, \& Prades, 2018; Van Deursen, Bolle, Hegner, \& Kommers, 2015).

El FoMO se define como el sentimiento de que a uno le falta información interesante o importante o que otras personas están haciendo algo divertido o interesante sin uno, lo que lleva a la frustración y la ansiedad asociadas con el sentimiento de quedarse fuera (Przybylski et al., 2013). Las redes sociales en línea a menudo crean esta experiencia porque hay un flujo constante de información que se produce sobre ellos en todo momento y porque los usuarios de redes sociales publican los aspectos más destacados de sus vidas. La conciencia de que siempre está sucediendo algo en línea, de que se comparte información interesante que otros conocen, pero tú no, crea ansiedad y una necesidad de "estar al tanto" para no quedar fuera. Esto causa una verificación frecuente, a menudo compulsiva, para mantenerse actualizado sobre lo que está sucediendo. Una vez en línea, los usuarios ven todas las cosas interesantes que están haciendo sus compañeros y comparan, consciente o inconscientemente, estas experiencias con sus propias vidas, lo que puede conducir a la insatisfacción con el yo (Baker \& Algorta, 2016; Gil, del Valle, Oberst, \& Chamarro, 2015; 
Przybylski, Murayama, Dehaan, \& Gladwell, 2013). Para muchas personas, también crea una necesidad competitiva de "ponerse al día" y alcanzar el nivel percibido de sus compañeros.

La búsqueda de validación en línea es el acto de publicar fotos halagadoras, actualizaciones de logros $u$ otro contenido que atraiga la atención en busca de comentarios positivos. La búsqueda de validación es problemática porque basa el valor de una persona y de sus experiencias en lo que otros piensan, es decir, la valoración deja de ser intrínseca (proviene del interior del ser) y comienza a ser extrínseca (depende de las percepciones de los demás). Cuando el valor es extrínseco, es inestable y transitorio y puede conducir a una búsqueda constante de más y más validación para permanecer en un estado afectivo positivo lo que puede estar relacionado con algunos rasgos de personalidad (Casale \& Banchi, 2020; Ross et al., 2009).

Evitar el afrontamiento de algunas situaciones es la última de las motivaciones asociada al uso problemático. Utilizar estrategias de evitación es el acto de distraerse de un estado interno desagradable o una situación externa en lugar de abordarlo y tratarlo directamente. Hacer frente al afrontamiento a través de la tecnología puede ser un problema leve, como cuando las personas recurren a sus teléfonos inteligentes para evitar el estado aversivo de aburrimiento, o puede ser más perjudicial, como cuando las personas usan la tecnología para evitar los sentimientos de ansiedad, el estrés de la depresión (Holahan, Moos, Holahan, Brennan, \& Schutte, 2005; Panova \& Lleras, 2016). Se ha sugerido que, con la disponibilidad constante de entretenimiento sin fin que permite la tecnología, las personas se han acostumbrado a estar siempre estimuladas cognitivamente y no se permiten experimentar estados de "tiempo de inactividad cognitivo" que son necesarios para la reflexión, el procesamiento emocional y descanso mental (Immordino-Yang, Christodoulou, \& Singh, 2012; Pang, 2013). El cerebro del usuario intensivo de redes sociales o de internet puede perder su capacidad de estimularse a sí mismo debido a la disponibilidad constante de estimulación externa.

Las personas también tienden a usar la tecnología para escapar de estados negativos aversivos como el estrés. En estos días, cuando una persona se encuentra en una situación incómoda, es típico que mire hacia a sus teléfonos inteligentes y se distraiga usando una red social para intentar desconectarse de la experiencia aversiva. Si bien hacerlo es un reflejo normal, ignorar un problema cierra la posibilidad de aprender de él y desarrollar habilidades de afrontamiento activas que pueden emplearse para lidiar con los estresores en el futuro. Los pequeños factores estresantes de la vida son campos de entrenamiento para la resiliencia y las habilidades de resolución de problemas que nos ayudan a manejar los factores estresantes más grandes. Hacer frente a la evitación a través de la tecnología ha demostrado ser un comportamiento potencialmente problemático (Kircaburun \& Griffiths, 2018) y se ha asociado con ansiedad y depresión (Panova \& Lleras, 2016) lo que nos hace pensar que sea un potencial mecanismo de afrontamiento desadaptativo (Blalock \& Joiner, 2000; Holahan et al., 2005).

\section{c. Adicción al teléfono móvil}

La evolución del uso de Internet y de los hábitos de acceso evidencia una nueva realidad. Por ejemplo, en el año 2015, en España se observó una supremacía clara del teléfono inteligente $(88,2 \%)$ sobre el ordenador $(78,2 \%)$ como dispositivo para acceder a Internet, sobre todo en las edades comprendidas entre los 14 y los 19 años. Esta evolución se observó también en la utilización del teléfono que superó al ordenador como dispositivo de acceso al ocio, lo que circunscribe la hegemonía del ordenador al ámbito profesional y educativo (Fundación Telefónica, 2016). Debido a su popularidad y a tratarse de un dispositivo relativamente nuevo, el teléfono inteligente (smartphone) ha despertado la preocupación por su potencial adictivo (Chóliz, Villanueva, \& Chóliz, 2009; Pedrero, Rodríguez, \& Ruiz, 2012). Desde 
nuestro punto de vista, la preocupación por la adicción al teléfono móvil ha tenido dos oleadas (Carbonell et al., 2018). En la primera, centrada en el teléfono móvil no inteligente, la preocupación se debía principalmente a dos factores, al importe de las facturas y al uso de los mensajes de texto. El importe de las facturas preocupaba porque no existía la tarifa plana y los usuarios necesitaron un periodo de aprendizaje para gestionar su uso y el importe de las facturas. Por su parte, los mensajes de texto permitían expresar emociones de forma espontánea, menos invasiva y menos comprometida emocionalmente que el 'cara a cara'. Cuando gracias a los sistemas de tarificación se aprendió a controlar el gasto y parecía que la preocupación disminuía, se lanzaron al mercado los teléfonos inteligentes iniciándose una nueva oleada sobre el posible uso adictivo de este dispositivo, en este caso vinculada al uso de Internet en general y de algunas de sus aplicaciones como redes sociales o servicios de mensajería (Carbonell, 2020c).

A esta preocupación hay que añadir que los medios de comunicación se hacen eco y tienden a propagar las informaciones negativas sobre el uso de móvil con mayor o menor fundamento científico. Estos fenómenos no se consideran adicciones comportamentales y se estudian dentro del amplio rango de trastornos que puede causar el uso del telefóno y de la tecnología. Son ejemplos de estas alertas el technostress, el smombie (una combinación de "smartphone" y "zombie"), el fear of missing out (fomo), la nomofobia ("no-mobile-phone phobia") y el phubbing (menospreciar o ignorar a quien nos acompaña por prestar mayor atención al teléfono) (Panova \& Carbonell, 2018).

Nuestras investigaciones apoyan la idea de que la percepción de uso problemático del móvil ha aumentado en la última década y que esta preocupación parece deberse al acceso a Internet y con ello a redes sociales y otras aplicaciones. Este uso problemático es específico y no general, es decir, depende de la actividad concreta que se lleve a cabo con el móvil y en Internet. Las aplicaciones del móvil más usadas por jóvenes y adolescentes son el correo electrónico y la mensajería, participar en redes sociales, escuchar música y jugar a videojuegos más o menos simples (Carbonell et al., 2018).

Sin embargo, pese a la existencia de este uso problemático parece que el término 'adicción' es un constructo inadecuado para referirnos al móvil porque, entre otras cosas, la conducta problemática no depende del móvil o de Internet sino de las aplicaciones que se utilicen (Panova \& Carbonell, 2018). Por ejemplo, apostar es un comportamiento que puede ser problemático independientemente de si se apuesta en un salón de apuestas o si se hace desde el móvil. Al igual que ha pasado con Internet, se tiende a confundir el alcohol con el vaso o la botella que lo contienen (Panova \& Carbonell, 2018) y, de cara a investigaciones futuras será más productivo estudiar los usos y actividades que se llevan a cabo con el teléfono en lugar del tiempo o la frecuencia de uso del teléfono (o del ordenador, la tableta o Internet) (Carbonell et al., 2018; Lowe-Calverley \& Pontes, 2020).

En la mayoría de los casos, el uso problemático de móvil que se obtiene mediante el uso de cuestionarios no se ha validado con medidas conductuales (Ellis, Davidson, Shaw, \& Geyer, 2019). Así, se han publicado muchas escalas para evaluar la adicción a teléfonos inteligentes (Csibi, Griffiths, Cook, Demetrovics, \& Szabo, 2018; Kim, Lee, Lee, Nam, \& Chung, 2014; Kwon et al., 2013), pero se desconoce si están refrendadas objetivamente entre otros factores porque no se corresponden con medidas objetivas de uso de móvil. Estos cuestionarios permiten detectar una preocupación y una percepción, pero en ningún caso emitir un diagnóstico clínico. Por otra parte, nos encontramos ante una ausencia de demanda clínica, en todo caso se observa la petición de padres que consideran que sus hijos adolescentes y preadolescentes tienen un uso inadecuado pero esta demanda no es necesariamente indicativa de un trastorno mental y el problema puede ser abordado desde una perspectiva exclusivamente psicoeducativa (Panova \& Carbonell, 2018). 
Los jóvenes utilizan el móvil cada vez más frecuentemente para consultar Internet y tienden a no distinguir entre la plataforma (móvil, ordenador) y el programa o app. Desde nuestro punto de vista, la rapidez con la que evoluciona la tecnología nos obliga a cambiar constantemente las preguntas de investigación. Recurrimos a una anécdota para ilustrar la situación. Una estudiante que en el año 2018 cursaba cuarto curso de psicología cuando se le mostró una foto de un teléfono Nokia 3210 original nos preguntó: "Pero ... ¿se podía ser adicto a eso?". Desde su perspectiva no era posible que una persona desarrollará una adicción a un dispositivo telefónico que carecía de Internet y, por tanto, no permitía acceder a redes sociales, juegos, etc.". Esta pregunta ingenua revela hasta qué punto el diagnóstico de las adicciones tecnológicas y, presumiblemente, el de otros trastornos mentales están influidos por la cultura.

Lo que no se puede negar es que el teléfono inteligente ha conseguido que muchas conductas sean más accesibles. Si antes se debía esperar a llegar a casa para consultar las redes sociales en el ordenador ahora es posible, llevarlo a cabo inmediatamente, en la absoluta privacidad y en una gran variedad de lugares. De alguna forma se puede comparar el móvil, con el cigarrillo. El formato cigarrillo es un producto industrial de los más exitosos cuyo precio, disponibilidad, facilidad de uso, etc. ha popularizado el consumo de nicotina donde ni el puro ni la pipa ni el rapé podrían haber llegado. Sin embargo, el cigarrillo no es el problema, sí lo es la nicotina. Es decir, el teléfono inteligente incrementa el riesgo adictivo de algunas conductas, pero no es el problema en sí mismo.

Aceptar una supuesta adicción a móvil, no reconocida como tal en los manuales psiquiátricos, implicaría reconocer como enfermedad una conducta que puede ser excesiva y ocasionar consecuencias negativas leves, pero es contraproducente 'patologizar' la vida cotidiana y considerar los excesos, malos hábitos de salud y las aficiones como una enfermedad, sobre todo en el caso de los más jóvenes (Petry \& O'Brien, 2013). Esta 'etiqueta' podría acompañarlos toda la vida cuando no tiene otro significado que tratarse de una conducta propia de una etapa evolutiva.

\section{d. El trastorno de juego en Internet}

Pese a que el trastorno de juego en Internet, o Internet gaming disorder (IGD) está recogida en la sección III del DSM-5 (American Psychiatric Association, 2013) y en la CIE-11 (World Health Organization, 2018) todavía no se ha alcanzado el pleno consenso entre los investigadores sobre su diagnóstico y tratamiento (Aarseth et al., 2017; Billieux, Castro, \& et al., 2021; Griffiths, Kuss, Lopez-Fernandez, \& Pontes, 2017; Griffiths et al., 2016; KardefeltWinther, 2015) aunque, sin duda, su inclusión en los manuales diagnósticos va a permitir mejorarlo (Montag et al., 2019; Petry \& O'Brien, 2013).

Para la CIE-11, el trastorno por uso de videojuegos (gaming disorder) se caracteriza por un patrón de comportamiento de juego persistente o recurrente ("juegos digitales" 0 "videojuegos"), que puede ser en línea (es decir, por Internet) o fuera de línea. Según la Organización Mundial de la Salud el trastorno por uso de videojuegos se caracteriza por: a) deterioro en el control sobre el juego (por ejemplo, inicio, frecuencia, intensidad, duración, terminación, contexto); b) incremento en la prioridad dada al juego al grado que se antepone a otros intereses y actividades de la vida diaria; y c) continuación o incremento del juego a pesar de que tenga consecuencias negativas. Al igual que el DSM-5, requiere un período de al menos 12 meses para que se asigne el diagnóstico.

A nuestro modo de ver, se han de tener en cuenta una serie de consideraciones en torno al trastorno de juego en Internet, del mismo tipo que cuando se analiza la posible adicción a las redes sociales y al teléfono móvil. En primer lugar, es necesario que las consecuencias sean graves y valorar su gravedad. Las consecuencias negativas tienen que ir más allá de una 
noche sin dormir, una discusión familiar o un bajón en el rendimiento escolar. A diferencia de lo que ocurre con el trastorno por consumo de sustancias, el DSM-5 no establece una gradación entre leve, moderado o grave. Esta gradación sería especialmente importante en una conducta en la que el continuum normalidad - patología es evidente y podría ser un primer paso para distinguir entre los jugadores por entretenimiento, con alto compromiso, problemáticos, adictos y profesionales, tal como ya sugirieron Charlton y Danforth (2007, 2010). Obviamente, un principiante de un videojuego como League of Legends necesita un período de entrenamiento y práctica para convertirse en profesional. ¿Cómo debemos referirnos a este período de tiempo: ¿alto compromiso?, ¿uso problemático?, ¿preprofesional?, ¿aficionado?, ¿jugador profesional? Pese a que el DSM-5 y la CIE-11 están pensados para adultos se corre el riesgo de aplicar preferiblemente el diagnóstico a adolescentes con el riesgo que una etiqueta diagnóstica puede marcar el desarrollo de un adolescente que transitoriamente se implica en una conducta que desaparecerá o remitirá espontáneamente. Hay que ser muy cautos antes de establecer un diagnóstico mental que acompañara a este adolescente a lo largo de su vida y que puede ser más incapacitante y estigmatizante que el propio trastorno.

La segunda consideración, es que las consecuencias negativas son más importantes que el tiempo que se invierte en jugar (Charlton \& Danforth, 2007, 2010; Wood \& Griffiths, 2007) aunque el tiempo dedicado a jugar impide el desarrollo de otras actividades y puede provocar consecuencias negativas de tipo físico, psicológico y/o social (Snodgrass, Lacy, Dengah, Fagan, \& Most, 2011). De hecho, en los criterios del DSM-5 y de la CIE-11 tiempo de juego no es un criterio diagnóstico, algo similar a lo que ocurre con los criterios para el diagnóstico del trastorno por consumo de sustancias que no recogen la cantidad de sustancia consumida.

En tercer lugar, es importante resaltar que se corre el riesgo de 'patologizar' a millones de video jugadores en todo el mundo (Griffiths et al., 2017; Kardefelt-Winther, 2015), igual que podría suceder con el móvil y con las redes sociales. Por ejemplo, la onicofagia es un hábito inadecuado con consecuencias perjudiciales (Ghanizadeh \& Shekoohi, 2011; Tanaka, Vitral, Tanaka, Guerrero, \& Camargo, 2008) que comparte algunos síntomas de una adicción conductual (por ejemplo, pérdida de control e incapacidad de abstinencia) pero, a falta de consecuencias graves, no se considera un trastorno mental. Un caso similar podría ser la 'adicción al chocolate' que no conviene equiparar a la de las sustancias que alteran el sistema nervioso central (Petry \& O'Brien, 2013). Una vez más, no conviene patologizar la vida cotidiana (Billieux, Schimmenti, Khazaal, Maurage, \& Heeren, 2015; Frances \& Widiger, 2012; Petry \& O'Brien, 2013).

También se ha apuntado que los adolescentes diagnosticados de IGD tienen más riesgo de estar diagnosticados de depresión y de conducta desafiante (Jo et al., 2019) o de retirada social (Matalí et al., 2020). Hasta el momento, no se ha reportado comorbilidad con consumo o adicción a drogas (Marcos \& Chóliz, 2020). Es importante estar atento a otros posibles trastornos primarios o secundarios. Ligado a la comorbilidad está el reto de establecer tipologías de adictos a las que llegaremos cuando avancemos en la comprensión del trastorno (Jo et al., 2019; Martín-Fernández et al., 2016; Matalí et al., 2020; TorresRodríguez, Griffiths, Carbonell, Farriols-Hernando, \& Torres-Jimenez, 2019).

Otro elemento a tener en cuenta es la evolución de los videojuegos. Pese a que la OMS considera que la adicción fuera de línea es posible, cada vez se hace más difícil entender un videojuego que no sea en línea, en el que no se interactúe durante el juego, se comente el ranking en redes sociales o se visione en streaming (televisión por Internet que retransmite acontecimientos frecuentemente deportivos o musicales, en directo o en diferido). Un videojuego offline tiene un principio y un final y los diseñadores calculan el tiempo que un jugador medio necesita invertir para 'superar las pantallas'. En estos videojuegos, independientemente de su modalidad, el jugador acostumbra a jugar en solitario, contra una 
inteligencia artificial, o en multijugador, en partidas cooperativas o competitivas, que suelen aglutinar entre dos y 64 jugadores. Estallo et al. (2001) analizaron el potencial adictivo de estos juegos y concluyeron que era bajo. Sin embargo, la capacidad adictiva de los videojuegos en línea es mayor. Por ejemplo, los Massively Multiplayer Online Role-Playing Games (MMORPG) presentan mundos complejos y persistentes cohabitados por miles de usuarios. En estos mundos la interacción con otros jugadores es el mejor modo de progresar $y$, en consecuencia, tienen sistemas específicos que rigen la formación de clanes con los mismos objetivos que implican una presión social para jugar (Carbonell, Torres-Rodríguez, \& Fuster, 2016).

Es importante tener presente que estas consideraciones se emiten en un contexto en el que pesa la relevancia económica de la industria, su popularidad y visibilidad (Carbonell, 2020b). Ejemplos de ello son el eco que se hizo en los medios de comunicación del premio de tres millones de dólares que ganó en julio de 2019, Kyle Giersdorf, un videojugador de 16 años (Del Palacio, 2019), las retransmisiones en streaming de la Liga de Videojuegos Profesional, la construcción de estadios específicos en Las Vegas o el nombramiento como deporte olímpico de exhibición (Redacción Marca, 2019). En este marco de poderío económico y capacidad de presión social, es conveniente repensar el modelo de 'jugador responsable' o 'bebedor responsable' que la industria construyó para los juegos de azar y el consumo de alcohol.

Aquí, el papel de las instituciones deportivas puede ser trascendental. Conviene recordar a este respecto que el bridge y el ajedrez son deportes sin actividad física que pertenecen a la Association of IOC Recognised International Sports Federations (ARISF, 2020) una organización constituida y reconocida por el Comité Olímpico Internacional cuyos miembros son federaciones deportivas internacionales que no compiten en los Juegos Olímpicos. Además de los dos mencionados, encontramos también la pelota vasca, las carreras de orientación o el polo.

Por último, queremos destacar la relación entre los juegos y la inversión económica que algunos requieren. Ésta suele estar relacionada con compras que mejoran aspectos del juego y algunos autores evidencian su problemática puesto que pueden relacionarse con la conducta asociada al juego de azar. Hablamos de los juegos pay-to-play, play-to-win y fremium. En los juegos pay-to-play se paga por jugar, por mantener activa la cuenta o por jugar dentro de otro juego. En el modelo pay-to-win, más frecuente en los juegos casuales de móvil y redes sociales, se puede pagar para mejorar la experiencia o para ganar; es una forma de comprar el tiempo necesario para progresar (Carbonell, Torres-Rodríguez, et al., 2016; Miguel, 2019). Los juegos freemium (contracción en inglés de las palabras free y premium) son un modelo de videojuego que funciona ofreciendo servicios básicos gratuitos, mientras se cobra dinero por otros servicios más avanzados o especiales (por ejemplo, los disfraces del Fortnite). Estos micropagos o microtransacciones son de uso único y se tiene que pagar cada vez que se quiere añadir algún cambio. La relación con los juegos de azar está en la compra de cajas de botín, cofres o cajas sorpresa de contenido aleatorio (loot box tal como se conoce en inglés). Como dice su expresión, se paga por un contenido que puede ir desde la espada más buscada hasta la armadura más común. Por lo tanto, los juegos gratuitos a menudo no son completamente gratuitos (Fuster, 2020). Es en estos modelos donde las fronteras entre el gaming (videojuegos) y el gambling (juegos de apuestas) tienden a desvanecerse porque hay adultos y jóvenes que pueden gastar cantidades importantes de dinero real. 


\section{Discusión y recomendaciones finales}

En el DSM-5, se pueden subrayar dos hitos importantes para las adicciones conductuales. El primero es que incluyó el gambling disorder (juego -de azar- patológico en la traducción oficial al castellano) en el mismo nivel que las adicciones a sustancias. El DSM no había reconocido las adicciones conductuales como un trastorno mental; a este efecto, se puede recordar que en el DSM-IV y en el DSM-IV-TR se incluía el juego patológico en el apartado de "Trastornos del control de impulsos no clasificados en otros apartados". El segundo hito fue la inclusión del Internet gaming disorder (IGD) en la sección tercera, la de los diagnósticos que necesitan más investigación (p.795-798). Por su parte, la OMS dio un paso más y reconoció plenamente el gaming disorder (online $y$ offline) en la CIE-11. Sin embargo, los manuales de psiquiatría todavía son reacios a incorporar otras posibles adicciones conductuales y, entre ellas, las digitales. El paso del tiempo, y con él la evolución social y tecnológica, nos ayudará a discriminar si la preocupación por estas conductas merecía o no la consideración de verdaderos trastornos 0 , sencillamente, estamos viviendo un periodo de adaptación (como le sucedió al Quijote con la imprenta).

Uno de estos cambios es el que se está produciendo sobre el propio concepto de adicción. Es de sobras aceptado que lo que opina la ciencia sobre el consumo de sustancias y sus consecuencias para la salud se basa por una parte en el conocimiento técnico y, por otra, en el consenso social. En este sentido, la elección de determinados términos es importante, pues sabemos que el lenguaje refleja la construcción social del fenómeno. Así, el DSM ya cambió el término 'substance' por el de 'drug' por ser más neutro y no implicar ni legalidad ni normalidad sobre la sustancia. Por su parte, el término 'adicción' implica una posición más psicológica que el de 'drogodependencia' que pone el énfasis en la droga o sustancia y no en el comportamiento adictivo. El lenguaje formal y coloquial también refleja parte de este debate. Así, la RAE entiende que una drogodependencia es el 'uso habitual de estupefacientes al que el drogadicto no se puede sustraer' mientras que la primera acepción de la palabra adicción es 'dependencia de sustancias o actividades nocivas para la salud o el equilibrio psíquico' y la segunda la 'afición extrema a alguien o algo'. Por tanto, según la RAE, se puede ser adicto a una sustancia, a una actividad o a una persona y podría tratarse tanto de un trastorno como de una afición (extrema, eso sí). Nuestra posición al respecto es clara, para que una conducta digital se considere una adicción las consecuencias tienen que ser graves. No tiene sentido utilizar el término adicción para describir una afición o una conducta con consecuencias negativas leves y es conveniente no confundir los términos del lenguaje coloquial con los del científico.

Tampoco debemos olvidar que, en un campo supuestamente bien establecido como es el del abuso de sustancias, todavía es controvertido si la cafeína además de producir intoxicación y abstinencia es capaz de generar un trastorno adictivo. Por eso, el trastorno por consumo de cafeína aparece en la Sección 3 del DSM-5 ("Condiciones para estudios posteriores"), justo en las páginas previas al IGD (American Psychiatric Association, 2013, p. 792). Y eso que conocemos desde hace muchos años los efectos de la cafeína y se han podido observar sus efectos en multitud de personas y situaciones (Addicott, 2014; Budney \& Emond, 2014). Si la cafeína todavía genera estas dudas, es lógico que tampoco se haya llegado al consenso con las adicciones digitales (Griffiths et al., 2017, 2016).

La perspectiva de género es importante en las tres conductas tecnológicas. Las mujeres utilizan más las redes sociales y el teléfono móvil. Los hombres juegan más a videojuegos, apuestan más y consultan más páginas de contenido pornográfico. En todo caso, el uso problemático de redes sociales de las mujeres es mayor, lo que puede deberse al papel que juegan en la comunicación y en el establecimiento de redes (Panova et al., 2020) y los hombres juegan más. Todo ello sin olvidar el interés de la industria para que la mujer se incorpore plenamente al universo de los videojuegos. 
Las redes sociales en línea, Internet y el teléfono móvil son, ante todo, herramientas para la interacción social. Dado que la interacción social es una necesidad humana fundamental, su popularidad es comprensible, especialmente entre los jóvenes que se encuentran en una etapa de desarrollo caracterizada por mayores necesidades sociales y de desarrollo de su identidad. En nuestra opinión, no se cumplen los criterios necesarios para respaldar que estas conductas sean verdaderos trastornos mentales porque sus consecuencias negativas no son lo suficientemente graves, las medidas para medir la "adicción" son en su mayoría autoinformes no estandarizados y no se han publicado estudios con muestras clínicas ni longitudinales. Los problemas que causan se pueden entender en el contexto del perfil psicológico del usuario, sus motivaciones y gratificaciones, su cultura, necesidades sociales y entorno, y las consecuencias a medio y largo plazo.

Por todo ello, parece que lo más sensato es orientar y educar sobre los usos adecuados de las tecnologías digitales en cuanto al tiempo invertido, el lugar donde se utiliza o los fines a los que se destina sin caer en el error de considerarlas una adicción. La adicción es una enfermedad muy grave que no debe confundirse con malos hábitos, excesos o aficiones.

Profundizar en una adecuada formación para padres y educadores en pautas de relación y habilidades sociales y (personales) parece la mejor respuesta a cualquier pregunta sobre las supuestas adicciones digitales, sus consecuencias y cómo superarlas. 


\section{Referencias}

Aarseth, E., Bean, A. M., Boonen, H., Colder Carras, M., Coulson, M., Das, D., ... Van Rooij, A. J. (2017). Scholars' open debate paper on the World Health Organization ICD-11 Gaming Disorder proposal. Journal of Behavioral Addictions, 6(3), 267-270. https://doi.org/10.1556/2006.5.2016.088

Addicott, M. A. (2014). Caffeine use disorder: A review of the evidence and future implications. Current Addiction Reports, 1(3), 186-192. https://doi.org/10.1007/s40429-014-0024-9

Aichner, T., Grünfelder, M., Maurer, O., \& Jegeni, D. (2020). Twenty-Five Years of Social Media: A Review of Social Media Applications and Definitions from 1994 to 2019. Cyberpsychology, Behavior, and Social Networking, 00(00), 1-8. https://doi.org/10.1089/cyber.2020.0134

American Psychiatric Association. (2013). Diagnostic and Statistical Manual of Mental Disorders (5th, ed ed.). Washington, DC USA: American Psychiatric Association.

Andreassen, C. S. (2015). Online Social Network Site addiction: A comprehensive review. Current Addiction Reports, 2(2), 175-184. https://doi.org/10.1007/s40429-015-0056-9

Andreassen, C. S., \& Pallesen, S. (2014). Social network site addiction - an overview. Current Pharmaceutical Design, 20(25), 4053-4061. https://doi.org/10.2174/13816128113199990616

ARISF. (2020). Association of IOC Recognised International Sport Federations. Retrieved January 29, 2020, from https://www.arisf.sport/

Baker, D. A., \& Algorta, G. P. (2016). The Relationship Between Online Social Networking and Depression: A Systematic Review of Quantitative Studies. Cyberpsychology, Behavior, and Social Networking, 19(11), 638-648. https://doi.org/10.1089/cyber.2016.0206

Billieux, J., Castro, J., \& et al. (2021). Expert appraisal of criteria for assessing gaming disorder: An international Delphi study. Addiction, Accepted.

Billieux, J., Schimmenti, A., Khazaal, Y., Maurage, P., \& Heeren, A. (2015). Are we overpathologizing everyday life? A tenable blueprint for behavioral addiction research. Journal of Behavioral Addictions, 4(3), 119-123. https://doi.org/10.1556/2006.4.2015.009

Blalock, J. A., \& Joiner, T. E. (2000). Interaction of cognitive avoidance coping and stress in predicting depression/anxiety. Cognitive Therapy and Research, 24(1), 47-65. https://doi.org/10.1023/ A: 1005450908245

Boyd, D. M. (2014). It's complicated: The social lives of networked teens. New Haven: Yale University Press.

Brien, C. P. O., Ph, D., Auriacombe, M., Bucholz, K., Ph, D., Budney, A., ... Ph, D. (2013). DSM-5 criteria for substance use disorders: Recommendations and rationale. American Journal of Psychiatry, 170 (August), 834-851.

Budney, A. J., \& Emond, J. A. (2014). Caffeine addiction? Caffeine for youth? Time to act! Addiction, 109(11), 1771-1772. https://doi.org/10.1111/add.12594

Calvo, F., Carbonell, X., Oberst, U., \& Fuster, H. (2018). May the passion be with you: The addictive potential of collectible card games, miniatures, and dice of the Star Wars universe. Journal of Behavioral Addictions, 7(3), 727-736. https://doi.org/10.1556/2006.7.2018.73

Carbonell, X. (2020a). Digitalidad y adicción: experiencias y reflexiones. Revista Española de Drogodependencias, 45(4), 9-13.

Carbonell, X. (2020b). El diagnóstico de adicción a videojuegos en el DSM-5 y en la CIE-11: retos y oportunidades para clínicos. Papeles Del Psicologo. https://doi.org/https://doi.org/10.23923/ pap.psicol2020.2935

Carbonell, X. (2020c). Potencial addictiu de lú's del telèfon mòbil. Perspectiva Escolar, 409(monográfico), $16-19$.

Carbonell, X., Chamarro, A., Oberst, U., Rodrigo, B., \& Prades, M. (2018). Problematic use of the Internet and smartphones in university students: 2006 - 2017. International Journal of Environmental Research and Public Health, 15(3), 1-13. https://doi.org/10.20944/ 
preprints201801.0236.v1

Carbonell, X., Guardiola, E., Beranuy, M., \& Bellés, A. (2009). A bibliometric analysis of the scientific literature on Internet, video games, and cell phone addiction. Journal of The Medical Library Association, 97(2), 102-107. https://doi.org/10.3163/1536-5050.97.2.006

Carbonell, X., Guardiola, E., Fuster, H., Gil, F., \& Panova, T. (2016). Trends in scientific literature on addiction to the internet, video games, and cell phones from 2006 to 2010. International Journal of Preventive Medicine, 7(1), 63. https://doi.org/10.4103/2008-7802.179511

Carbonell, X., \& Panova, T. (2017). A critical consideration of social networking sites' addiction potential. Addiction Research \& Theory, 25(1), 48-57. https://doi.org/10.1080/16066359.2016.1197915

Carbonell, X., Torres-Rodríguez, A., \& Fuster, H. (2016). El potencial adictivo de los videojuegos. In Abuso de Internet: cantesala para la adicción al juego de azar on-line? (pp. 83-103). Madrid: Pirámide.

Casale, S., \& Banchi, V. (2020). Narcissism and problematic social media use: A systematic literature review. Addictive Behaviors Reports, 100252. https://doi.org/10.1016/j.abrep.2020.100252

Charlton, J. P., \& Danforth, I. D. W. (2007). Distinguishing addiction and high engagement in the context of online game playing. Computers in Human Behavior, 23, 1531-1548. https://doi.org/10.1016/ j.chb.2005.07.002

Charlton, J. P., \& Danforth, I. D. W. (2010). Validating the distinction between computer addiction and engagement: Online game playing and personality. Behaviour \& Information Technology, 29(6), 601-613. https://doi.org/10.1080/01449290903401978

Chóliz, M. C., Villanueva, V., \& Chóliz, M. C. (2009). Ellas, ellos y su móvil: Uso, abuso (¿y dependencia?) del teléfono móvil en la adolescencia. Revista Española de Drogodependencias, 34(1), 74-88.

Csibi, S., Griffiths, M. D., Cook, B., Demetrovics, Z., \& Szabo, A. (2018). The psychometric properties of the Smartphone Application-Based Addiction Scale (SABAS). International Journal of Mental Health and Addiction, 16(2), 393-403. https://doi.org/10.1007/s11469-017-9787-2

Del Palacio, G. (2019, July 30). Kyle Giersdorf "Bugha", el millonario de 16 años que ganó el Mundial de Fortnite | Videojuegos. El Mundo. Retrieved from https://www.elmundo.es/tecnologia/videojuegos/ 2019/07/30/5d3f278021efa0641f8b4637.html

Ellis, D. A., Davidson, B. I., Shaw, H., \& Geyer, K. (2019). Do smartphone usage scales predict behavior? International Journal of Human Computer Studies, 130, 86-92. https://doi.org/10.1016/ j.ijhcs.2019.05.004

Estallo, J. A., Masferrer, M. C., \& Aguirre, C. (2001). Efectos a largo plazo del uso de videojuegos. Apuntes de Psicología, 19(1), 161-174.

Frances, A. J., \& Widiger, T. (2012). Psychiatric diagnosis: Lessons from the DSM-IV past and cautions for the DSM-5 future. Annual Review of Clinical Psychology, 8, 109-130. https://doi.org/10.1146/ annurev-clinpsy-032511-143102

Fundación Telefónica. (2016). La sociedad de la información en España 2016. Barcelona: Fundación Telefónica.

Fuster, H. (2020). El diseño de videojuegos: diversión y pitencial adictivo como dos caras de la misma moneda. Revista Española de Drogodependencias, 45(4), 49-64.

Ghanizadeh, A., \& Shekoohi, H. (2011). Prevalence of nail biting and its association with mental health in a community sample of children. BMC Research Notes, 4(1), 116. https://doi.org/ 10.1186/1756-0500-4-116

Gil, F., del Valle, G., Oberst, U., \& Chamarro, A. (2015). Nuevas tecnologías - ¿ Nuevas patologías? El smartphone y el fear of missing out. Aloma, 33(2), 77-83.

Goodman, M. D. (1990). Addiction: Definition and implications. British Journal of Addictions, 85(1), 1403-1408. https://doi.org/10.1111/j.1360-0443.1990.tb01620.x 
Griffiths, M. D. (1995). Technological addictions. Clinical Psychology Forum, 76, 14-19.

Griffiths, M. D. (2005). A 'components' model of addiction within a biopsychosocial framework. Journal of Substance Use, 10, 191-197. https://doi.org/10.1080/14659890500114359

Griffiths, M. D., Kuss, D. J., Lopez-Fernandez, O., \& Pontes, H. M. (2017). Problematic gaming exists and is an example of disordered gaming. Journal of Behavioral Addictions, 6(3), 296-301. https:// doi.org/10.1556/2006.6.2017.037

Griffiths, M. D., van Rooij, A. J., Kardefelt-Winther, D., Starcevic, V., Király, O., Pallesen, S., ... Demetrovics, Z. (2016). Working towards an international consensus on criteria for assessing internet gaming disorder: A critical commentary on Petry et al. (2014). Addiction, 111(1), 167175. https://doi.org/10.1111/add.13057

Holahan, C. J., Moos, R. H., Holahan, C. K., Brennan, P. L., \& Schutte, K. K. (2005). Stress Generation, Avoidance Coping, and Depressive Symptoms: A 10-Year Model. Journal of Consulting and Clinical Psychology, 73(4), 658-666. https://doi.org/10.1037/0022-006X.73.4.658

Immordino-Yang, M. H., Christodoulou, J. A., \& Singh, V. (2012). Rest Is Not Idleness. Perspectives on Psychological Science, 7(4), 352-364. https://doi.org/10.1177/1745691612447308

Jeno, L. M., Vandvik, V., Eliassen, S., \& Grytnes, J.-A. (2019). Testing the novelty effect of an m-learning tool on internalization and achievement: A Self-Determination Theory approach. Computers \& Education, 128, 398-413. https://doi.org/https://doi.org/10.1016/j.compedu.2018.10.008

Jo, Y. S., Bhang, S. Y., Choi, J. S., Lee, H. K., Lee, S. Y., \& Kweon, Y.-S. (2019). Clinical characteristics of diagnosis for Internet Gaming Disorder: Comparison of DSM-5 IGD and ICD-11 GD diagnosis. Journal of Clinical Medicine, 8(7), 945. https://doi.org/10.3390/jcm8070945

Kardefelt-Winther, D. (2015). A critical account of DSM-5 criteria for internet gaming disorder. Addiction Research \& Theory, 23(2), 93-98. https://doi.org/10.3109/16066359.2014.935350

Kardefelt-Winther, D., Heeren, A., Schimmenti, A., van Rooij, A., Maurage, P., Carras, M., ... Billieux, J. (2017). How can we conceptualize behavioural addiction without pathologizing common behaviours? Addiction, 112(10), 1709-1715. https://doi.org/10.1111/add.13763

Kim, D., Lee, Y., Lee, J., Nam, J. K., \& Chung, Y. (2014). Development of Korean Smartphone Addiction Proneness Scale for youth. PLOS ONE, 9(5). https://doi.org/10.1371/journal.pone.0097920

Kircaburun, K., \& Griffiths, M. D. (2018). Instagram addiction and the Big Five of personality: The mediating role of self-liking. Journal of Behavioral Addictions, 7(1), 158-170. https://doi.org/ $10.1556 / 2006.7 .2018 .15$

Kuss, D. J., \& Griffiths, M. D. (2011). Online social networking and addiction - A review of the psychological literature. International Journal of Environmental Research and Public Health, 8(9), 3528-3552. https://doi.org/10.3390/ijerph8093528

Kwon, M., Lee, J. Y., Won, W. Y., Park, J. W., Min, J. A., Hahn, C., ... Kim, D. J. (2013). Development and validation of a Smartphone Addiction Scale (SAS). PLOS ONE, 8(2). https://doi.org/10.1371/ journal.pone.0056936

Lowe-Calverley, E., \& Pontes, H. M. (2020). Challenging the concept of smartphone addiction: An empirical pilot study of smartphone usage patterns and psychological well-being. Cyberpsychology, Behavior, and Social Networking, 23(8), 550-556. https://doi.org/10.1089/cyber.2019.0719

Marcos, M., \& Chóliz, M. (2020). Comorbilidad entre adicciones comportamentales en la adolescencia. Revista Española de Drogodependencias, 45(4), 86-95.

Martín-Fernández, M., Matalí, J. L., García-Sánchez, S., Pardo, M., Lleras, M., \& Castellano-Tejedor, C. (2016). Adolescentes con trastorno por juego en Internet (IGD): perfiles y respuesta al tratamiento. Adicciones, $x x(\mathrm{x}), 125-133$. https://doi.org/10.20882/adicciones. 890

Matalí, J. L., Pérez-Racana, A., Oberst, U., Lleras, M., Bertomeu, P., \& Flores, E. (2020). Seclusion in adolescents with IGD: New diagnostic criteria or measure of severity? Computers in Human Behavior, 110(July 2019). https://doi.org/10.1016/j.chb.2020.106394

Miguel, E. (2019). Qué son las loot boxes de los juegos modernos y por qué las acusan de fomentar la 
ludopatía en los chavales. Retrieved from Magnet- Xataka website: https://magnet.xataka.com/ preguntas-no-tan-frecuentes/que-son-las-loot-boxes-de-los-juegos-modernos-y-por-que-lasacusan-de-fomentar-la-ludopatia-en-los-chavales

Montag, C., Schivinski, B., Sariyska, R., Kannen, C., Demetrovics, Z., \& Pontes, H. M. (2019). Psychopathological symptoms and gaming motives in Disordered Gaming - A psychometric comparison between the WHO and APA diagnostic frameworks. Journal of Clinical Medicine, 8(1691), 1-18. https://doi.org/doi:10.3390/jcm8101691

Pang, A. S.-K. (2013). The Distraction Addiction. New York: Little Brown and Company.

Panova, T., \& Carbonell, X. (2018). Is smartphone addiction really an addiction? Journal of Behavioral Addictions, 7(2), 252-259. https://doi.org/10.1556/2006.7.2018.49

Panova, T., Carbonell, X., Chamarro, A., \& Puerta-Cortés, D. X. (2020). Specific smartphone uses and how they relate to anxiety and depression in university students: a cross-cultural perspective. Behaviour and Information Technology, 39(4), 944-956. https://doi.org/ 10.1080/0144929X.2019.1633405

Panova, T., \& Lleras, A. (2016). Avoidance or boredom: Negative mental health outcomes associated with use of Information and Communication Technologies depend on users' motivations. Computers in Human Behavior, 58, 249-258. https://doi.org/10.1016/j.chb.2015.12.062

Pedrero, E. J., Rodríguez, M. T., \& Ruiz, J. M. (2012). Adicción o abuso del teléfono móvil. Revisión de la literatura. Adicciones, 24(2), 139-152.

Petry, N. M., \& O'Brien, C. P. (2013). Internet gaming disorder and the DSM-5. Addiction, 108(7), 11861187. https://doi.org/10.1111/add.12162

Przybylski, A. K., Murayama, K., Dehaan, C. R., \& Gladwell, V. (2013). Motivational, emotional, and behavioral correlates of fear of missing out. Computers in Human Behavior, 29(4), 1841-1848. https://doi.org/10.1016/j.chb.2013.02.014

Redacción Marca. (2019, September 11). Los eSports se acercan a los Juegos Olímpicos de Tokio 2020. Marca. Retrieved from https://us.marca.com/claro/mas-deporte/ 2019/09/11/5d790ab622601d0f198b45eb.html

Ross, C., Orr, E. S., Sisic, M., Arseneault, J. M., Simmering, M. G., \& Orr, R. R. (2009). Personality and motivations associated with Facebook use. Computers in Human Behavior, 25(2), 578-586. https://doi.org/10.1016/j.chb.2008.12.024

Ryan, T., Chester, A., Reece, J., \& Xenos, S. (2014). The uses and abuses of Facebook: A review of Facebook addiction. Journal of Behavioral Addictions, 3(3), 133-148. https://doi.org/10.1556/ JBA.3.2014.016

Sánchez-Carbonell, X., Beranuy, M., Castellana, M., Chamarro, A., \& Oberst, U. (2008). La adiccion a Internet y al móvil: ¿Moda o trastorno? Adicciones, 20(2), 149-160.

Saunders, J. B. (2017). Substance use and addictive disorders in DSM-5 and ICD 10 and the draft ICD 11. Current Opinion in Psychiatry, 30(4), 227-237. https://doi.org/10.1097/ YCO.0000000000000332

Snodgrass, J. G., Lacy, M. G., Dengah, H. J. F., Fagan, J., \& Most, D. E. (2011). Magical flight and monstrous stress: Technologies of absorption and mental wellness in Azeroth. Culture, Medicine and Psychiatry, 35(1), 26-62. https://doi.org/10.1007/s11013-010-9197-4

Tanaka, O. M., Vitral, R. W. F., Tanaka, G. Y., Guerrero, A. P., \& Camargo, E. S. (2008). Nailbiting, or onychophagia: A special habit. American Journal of Orthodontics and Dentofacial Orthopedics, 134(2), 305-308. https://doi.org/10.1016/j.ajodo.2006.06.023

Torres-Rodríguez, A., Griffiths, M. D., Carbonell, X., Farriols-Hernando, N., \& Torres-Jimenez, E. (2019). Internet Gaming Disorder treatment: A case study evaluation of four different types of adolescent problematic gamers. International Journal of Mental Health and Addiction, 17(1). https://doi.org/ $10.1007 /$ s11469-017-9845-9

Turel, O., \& Bechara, A. (2016). Social networking site use while driving: ADHD and the mediating roles 
of stress, self-esteem and craving. Frontiers in Psychology, 7(MAR). https://doi.org/10.3389/ fpsyg.2016.00455

Turel, O., He, Q., Xue, G., Xiao, L., \& Bechara, A. (2014). Examination of neural systems sub-serving Facebook "Addiction." Psychological Reports, 115(3), 675-695. https://doi.org/ 10.2466/18.PR0.115c31z8

Van Deursen, A., Bolle, C. L., Hegner, S. M., \& Kommers, P. A. M. (2015). Modeling habitual and addictive smartphone behavior. The role of smartphone usage types, emotional intelligence, social stress, self-regulation, age, and gender. Computers in Human Behavior, 45, 411-420. https:// doi.org/10.1016/j.chb.2014.12.039

Wood, R., \& Griffiths, M. D. (2007). Time loss whilst playing video games: Is there a relationship to addictive behaviours? International Journal of Mental Health and Addiction, 5, 141-149.

World Health Organization. (2018). International Classification of Diseases 11th Revision 11th Revision (ICD-11). Geneva, Switzerland: World Health Organization. Retrieved from https://icd.who.int/

World Health Organization. (2019). International Classification of Diseases: ICD-11 for Mortality and Morbidity Statistics.

Young, K. S. (1996). Internet Addiction: The emergence of a new clinical disorder. 104th Annual Meeting of the American Psychological Association, 1(3), 237-244. Toronto, Canada. https://doi.org/ https://doi.org/10.1089/cpb.1998.1.237 